\title{
Big Bang Nucleosynthesis in Carmeli Cosmology-Mass Density, Temperature and Expansion Rate of the Early Universe
}

\author{
Firmin J. Oliveira \\ East Asian Observatory/James Clerk Maxwell Telescope, Hilo, USA \\ Email: firmjay@hotmail.com
}

How to cite this paper: Oliveira, F.J. (2021) Big Bang Nucleosynthesis in Carmeli Cosmology-Mass Density, Temperature and Expansion Rate of the Early Universe. Journal of High Energy Physics, Gravitation and Cosmology, 7, 333-343.

https://doi.org/10.4236/jhepgc.2021.71017

Received: September 14, 2020

Accepted: January 26, 2021

Published: January 29, 2021

Copyright $\odot 2021$ by author(s) and Scientific Research Publishing Inc. This work is licensed under the Creative Commons Attribution International License (CC BY 4.0).

http://creativecommons.org/licenses/by/4.0/

\section{(c) (i) Open Access}

\begin{abstract}
The Carmeli Cosmological Special Relativity theory (CSR) is used to study the universe at early times after the big bang. The universe temperature vs. time relation is developed from the mass density relation. It is shown that CSR is well suited to analyze the nucleosynthesis of the light elements up to beryllium, equivalent to the standard model.
\end{abstract}

\section{Keywords}

Big Bang Nucleosynthesis, Carmeli Cosmology, Nucleosynthesis

\section{Introduction}

Although Carmeli described the temperature of the early universe using his own Cosmological Special Relativity (CSR) theory [1] [2] [3], it was about an order of magnitude lower than the standard model. But closer study reveals that when proper account is taken for the internal degrees of freedom of the elementary particles at the epoch of nucleosynthesis, that the temperature vs. time relationship so obtained is equivalent with that of the standard model. This, by itself, would make the nucleosynthesis reactions analysis for CSR identical with the results of the standard model. In this regard, we will not carry out any light element synthesis since these have already been adequately explored [4].

\section{Mass Density}

In CSR, the redshift $z$ of light emitted in the past at time $t^{\prime}$ when the size of the universe had a radius of $R\left(t^{t}\right)$ is defined by

$$
1+z=\frac{R_{0}}{R\left(t^{\prime}\right)},
$$


where $R_{0}$ is the radius of the universe at the present epoch of time. It can be shown [5] that the relation for $z$ takes the form

$$
1+z=\sqrt{\frac{1+t^{\prime} / \tau}{1-t^{\prime} / \tau}}
$$

where $\tau$ is the Hubble-Carmeli time constant, the age of the universe in Carmeli cosmology. Combining (1) and (2), in CSR, a distance $R_{0}$ measured by an observer at the present epoch time $t^{\prime}=0$, transforms to the distance $R\left(t^{\prime}\right)$ for a time $t^{\prime}$ in the past given by

$$
R\left(t^{\prime}\right)=R_{0} \sqrt{\frac{1-t^{\prime} / \tau}{1+t^{\prime} / \tau}},
$$

with the limit into the past being the big bang time $t^{\prime}=\tau$. Note that in the Carmeli cosmological theory, time is measured from the present at $t^{\prime}=0$ toward the past at time $t^{\prime}>0$. The Hubble constant $H_{0} \approx 1 / \tau$. If we take $R_{0}=\varphi_{0}\left(t^{\prime}\right) c \tau$ to be the radial distance to the edge of the universe, where $\mathrm{c}$ is the speed of light in vacuum and $\varphi_{0}\left(t^{t}\right)$ is a scaling factor, then $R\left(t^{t}\right)$ from (3) is the universe radius at a past time $t^{\prime}$.

For nucleosynthesis studies it is conventional to measure time from the big bang toward the present. Transforming (3) by the change of variable $t=\tau-t^{\prime}$, we have for the universe radius

$$
R(t)=\frac{\phi_{0}(t) c \tau}{\sqrt{2 \tau / t-1}},
$$

where $t$ is the time measured from the big bang when $t=0$ toward the present when $t=\tau$ and the scaling factor

$$
\begin{aligned}
& \phi_{0}(t)=\frac{T_{0}}{T_{0 \text { past }}} \text { if } t<1.98 \times 10^{13} \text { sec., } \\
& \phi_{0}(t)=1 \text { otherwise. }
\end{aligned}
$$

where $T_{0} \approx 2.73 \mathrm{~K}$ is the present CMB temperature and $T_{0 \text { past }} \approx 14.42 \mathrm{~K}$ is the past radiation field temperature, for which we will give a derivation subsequently. Note that the cutoff time $t c=1.98 \times 10^{13} \mathrm{sec}$. is an approximate time representing the recombination time when the universe temperature was $0.3 \mathrm{eV}$, and this cutoff time should expect to be tweaked when studying that period.

The mass density $\rho(t)$ of the universe is defined to be composed of a relativistic mass density $\rho_{r}(t)$ and a non-relativistic mass density $\rho_{m}(t)$, where the total mass density $\rho(t)=\rho_{r}(t)+\rho_{m}(t)$.

We define the non-relativistic mass density in the usual way,

$$
\rho_{m}(t)=\frac{M_{m}}{V(t)},
$$

where $M_{m}$ is the total non-relativistic (rest) mass of the universe and $V(t)$ is the volume of the universe. The non-relativistic mass is defined by

$$
M_{m}=g_{t} M=g_{t} f_{m}\left(\frac{\tau c^{3}}{4 G}\right)
$$


where $M=f_{m} \tau c^{3} / 4 G$ is the total mass of the universe and $f_{m}$ and $g_{t}$ are coefficients which depend on the baryon to photon number density ratio $\eta$ and the baryon number density $n_{B}$ at the present time. Expressions for these constant coefficients will be shown to be given by

$$
\begin{gathered}
g_{t}=\frac{1}{\left(\zeta k_{B} T_{0} / \eta m_{p} c^{2}\right)+1}, \\
f_{m}=2 \Omega_{B}\left[\left(\zeta k_{B} T_{0} / \eta m_{p} c^{2}\right)+1\right],
\end{gathered}
$$

where $\zeta \approx 2.70$ is the black body average energy coefficient, $G$ is Newton's gravitational constant, $k_{B}$ is Boltzmann's constant, $T_{0} \approx 2.73 \mathrm{~K}$ is the present CMB temperature and the baryon density parameter $\Omega_{B}=m_{p} n_{B} / \rho_{c}$, where $m_{p}$ is the proton mass and $\rho_{c}=3 / 8 \pi G \tau^{2}$ is the critical mass density. We use the standard value $\tau=4.28 \times 10^{17} \mathrm{~s}$. For $\eta \approx 6.61 \times 10^{-10}$ and $\Omega_{B} \approx 0.046$, we have $g_{t} \approx 0.999$ and $f_{m} \approx 0.093$.

The relativistic mass is defined by

$$
M_{r}(t)=f_{m}\left(1-g_{t}\right)\left(\frac{\tau c^{3}}{4 G}\right)\left(\frac{c \tau}{R(t)}\right) .
$$

The volume for Euclidean flat space is given by

$$
V(t)=\frac{4}{3} \pi R^{3}(t)
$$

The non-relativistic mass density (6), using (7) and (11) is given by

$$
\rho_{m}(t)=\frac{M_{m}}{V(t)}=\left(\frac{f_{m} g_{t}}{\phi_{0}^{3}(t)}\right)\left(\frac{3}{16 \pi G \tau^{2}}\right)[(2 \tau / t)-1]^{3 / 2} .
$$

The relativistic mass density $\rho_{r}(t)$, using (10) and (11), is given by

$$
\rho_{r}(t)=\frac{M_{r}(t)}{V(t)}=\left(\frac{f_{m}\left(1-g_{t}\right)}{\phi_{0}^{4}(t)}\right)\left(\frac{3}{16 \pi G \tau^{2}}\right)[(2 \tau / t)-1]^{2} .
$$

The total mass density parameter is given by the sum of (12) and (13) divided by the critical density $\rho_{c}$

$$
\Omega(t)=\frac{\rho_{r}(t)+\rho_{m}(t)}{\rho_{c}}=\left[\frac{f_{m}\left(1-g_{t}\right)}{\phi_{0}^{4}(t)}\right][(2 \tau / t)-1]^{2}\left(1+\frac{\phi_{0}(t)\left[g_{t} /\left(1-g_{t}\right)\right]}{[(2 \tau / t)-1]^{1 / 2}}\right) .
$$

Figure 1 shows the total mass density parameter $\Omega(t)$ for the first 1000 seconds since the big bang. Using (8) and (9) in (14), the total mass density parameter at the present epoch $t=\tau$ takes on the value $\Omega(\tau)=\frac{f_{m}}{2}=\Omega_{B}\left[\left(\zeta k_{B} T_{0} / \eta m_{p} c^{2}\right)+1\right]$.

\section{Mass Density vs. Temperature}

Assuming that the universe expands adiabatically, the relativistic density $\rho_{s}(T)$ of the universe is related to the temperature by [6]. 


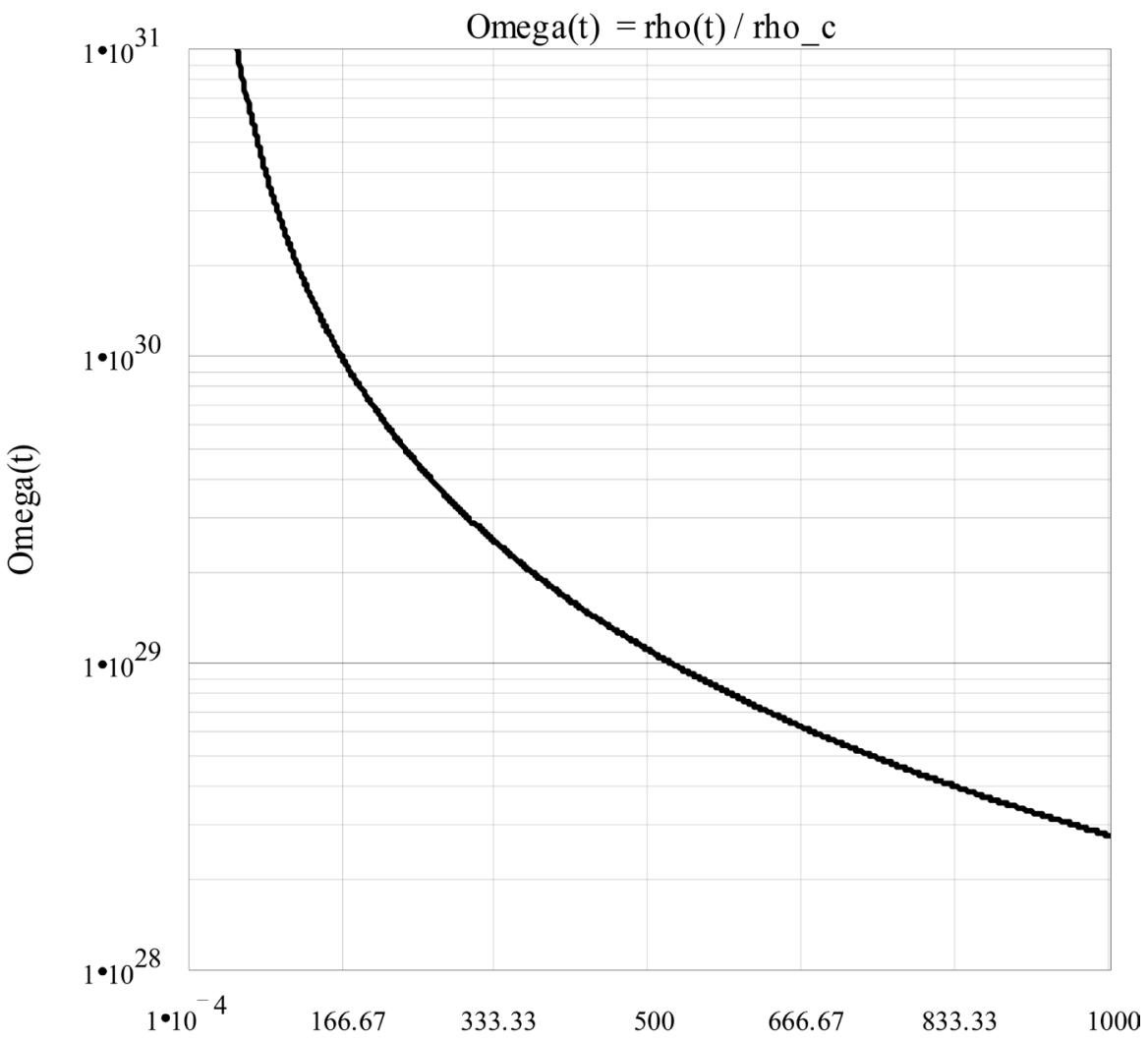

Figure 1. Total mass density parameter. The horizontal axis is time in seconds since the big bang.

$$
\rho_{s}(T)=\left(\frac{\pi^{2} g_{a}(T)}{30}\right) T^{4},
$$

where $g_{a}(T)$ is the effective number of degrees of freedom for the particles involved in the annihilation process at temperature $T$, where $T$ is in $\mathrm{MeV}$. Take the density (13), scale it and convert it from ergs to $\mathrm{MeV}$ and equate it to (15), yielding,

$$
\left(\frac{\zeta \mathrm{MeV}}{8}\right)\left(\frac{\phi_{0}^{4}(t)}{f_{m}\left(1-g_{t}\right)}\right) \rho_{r}=\left(\frac{3 \zeta \mathrm{MeV}}{32 \pi G}\right)\left(\frac{1}{t}-\frac{1}{2 \tau}\right)^{2}=\left(\frac{\pi^{2} g_{a}(T)}{30}\right) T^{4},
$$

where $\mathrm{MeV} \approx 6.24 \times 10^{5} \mathrm{MeV} \cdot \mathrm{erg}^{-1}$. Equation (16) can be put into the form of time (seconds) since the big bang as a function of temperature $(\mathrm{MeV})$,

$$
t(T)=\frac{2 \tau}{2 \tau\left(\sqrt{16 \pi^{3} G g_{a}(T) / 45}\right)(\sqrt{1 / \zeta M e V}) T^{2}+1} .
$$

Equation (16) can also be put into the form of temperature (MeV) as a function of time (seconds) since the big bang,

$$
T(t)=\left(\frac{45 \zeta \mathrm{MeV}}{16 \pi^{3} G g_{a}(T)}\right)^{1 / 4}\left(\sqrt{\frac{1}{t}-\frac{1}{2 \tau}}\right) .
$$

Setting $g_{a}(T)=3.363 \quad$ (after e+, e- annihilation when the temperature 
$T<500 \mathrm{keV}$ and the neutrinos have decoupled), setting $t=\tau$ and ignoring any further reactions such as the recombination period when the temperature was around $0.3 \mathrm{eV}$, (18) gives

$$
T_{0 \text { past }} T(\tau) \approx 14.42 \mathrm{~K},
$$

which we used in (5).

Since $(1 / 2 \tau) \ll(1 / t)$ at early times, it can be neglected so that (18) can be written more simply

$$
T^{2}(t)=\left(\frac{2.4255}{\sqrt{3.363}}\right)\left(\frac{1}{t}\right)
$$

which is equivalent to the expression in [6], Eq. 43,

$$
T^{2}(t)=\left(\frac{2.4}{\sqrt{g_{a}}}\right)\left(\frac{1}{t}\right),
$$

for $g_{a}=3.363$. Figure 2 shows the temperature of the expanding universe for the first 100 seconds since the big bang. Figure 3 shows the temperature of the universe for the first 1000 seconds.

The equivalence of the Carmeli big bang temperature model (20) with the standard model (21), implies that light element reaction analysis using Carmeli's model will agree with the standard model nucleosynthesis results.

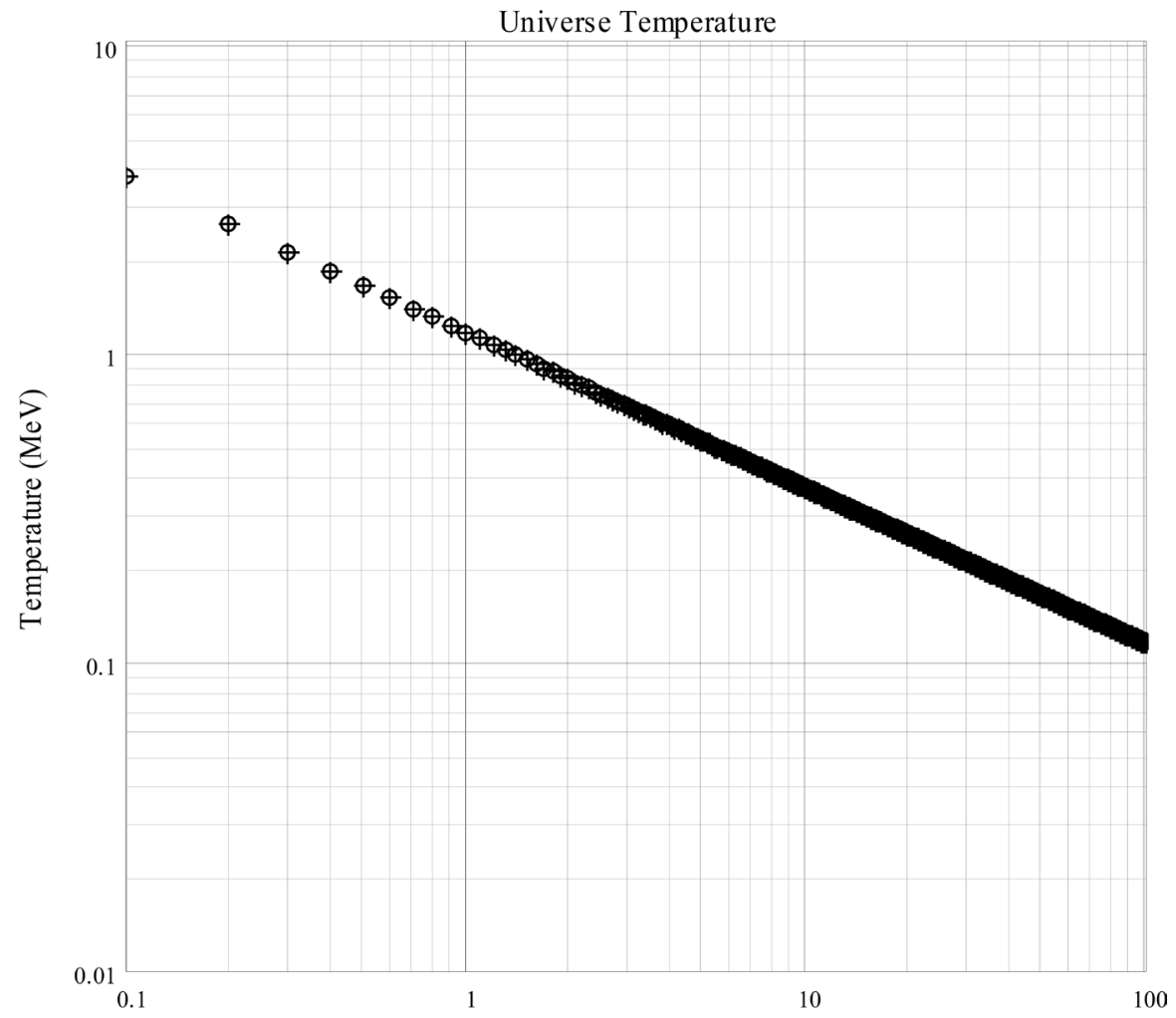

Figure 2. Temperature of the universe. The horizontal axis is time in seconds since the big bang. Open circles are for the standard model. Crosses are for the Carmeli model. 


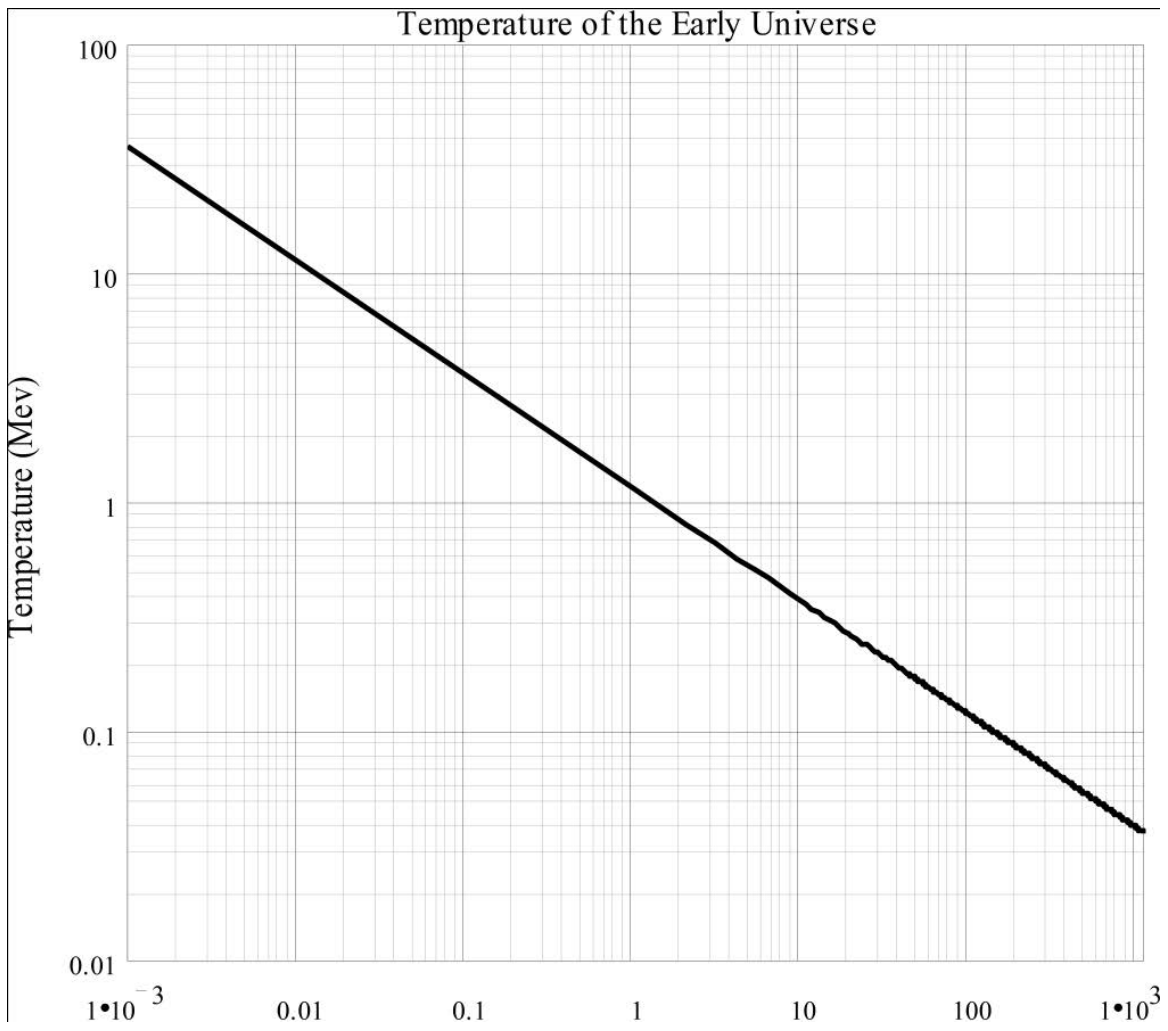

Figure 3. Temperature of the universe. The horizontal axis is time in seconds since the big bang.

\section{Expansion rate equation $H(t)$}

We now take the time rate of change $\mathrm{d} R(t) / \mathrm{d} t$ of the universe radius given by (4) relative to $R(t)$, obtaining the expansion rate $H(t)$,

$$
\begin{aligned}
H(t) & =\left(\frac{1}{R(t)}\right) \frac{\mathrm{d}}{\mathrm{d} t} R(t)=\left(\frac{1}{R(t)}\right) \frac{\mathrm{d}}{\mathrm{d} t}\left(\frac{R_{0}}{\sqrt{2 \tau / t-1}}\right) \\
& =\left(\frac{1}{2}\right)\left[\frac{1}{t}+\frac{1}{2 \tau-t}\right] \approx \frac{1}{2 t}
\end{aligned}
$$

where the approximation is good for $t \ll \tau$ as is the case for BBN. This is equivalent to the standard Friedmann-Lemaitre-Robertson-Walker expansion rate for Euclidean space (zero curvature) [7], implying that we are in agreement with the standard big bang model.

\section{Derivation of $f_{m}$ and $g_{t}$}

We derive expressions for $f_{m}$ and $g_{t}$ in terms of the baryon to photon number ratio $\eta(t)=n_{B}(t) / n_{\gamma}(t)$, where $n_{B}(t)$ is the baryon number density and $n_{\gamma}(t)$ is the photon number density, and where $n_{B}(t)=\rho_{m}(t) / m_{p}$, where $m_{p}$ is the proton rest mass and $n_{\gamma}(t)=\rho_{r}(t) c^{2} / \zeta k_{B} T(t)$, where $\zeta k_{B} T(t)$ is the average energy of the photons in the black body radiation field at temperature $T(t)$. Using (12) and (13) we have

$$
n_{B}(t)=\frac{\rho_{m}(t)}{m_{p}}=\left(\frac{f_{m} g_{t}}{\phi_{0}^{3}(t) m_{p}}\right)\left(\frac{3}{16 \pi G \tau^{2}}\right)[(2 \tau / t)-1]^{3 / 2},
$$




$$
\begin{aligned}
n_{\gamma}(t)=\frac{\rho_{r}(t) c^{2}}{\zeta k_{B} T(t)} & =\left[\frac{f_{m}\left(1-g_{t}\right)}{\phi^{3}(t) \zeta k_{B} T_{0}}\right]\left(\frac{3 c^{2}}{16 \pi G \tau^{2}}\right)[(2 \tau / t)-1]^{3 / 2}, \\
\eta & =\frac{n_{B}(t)}{n_{\gamma}(t)}=\frac{\zeta k_{B} T_{0} g_{t}}{m_{p} c^{2}\left(1-g_{t}\right)},
\end{aligned}
$$

where $T(t)$ is the temperature (K) at time $t$ since the big bang and is given by

$$
T(t)=T_{0}\left(\frac{c \tau}{R(t)}\right)=\left(\frac{T_{0}}{\phi_{0}(t)}\right) \sqrt{(2 \tau / t)-1},
$$

where $\phi_{0}(t)$ is given by (5).

From (24) we can solve for $g_{\text {p }}$

$$
g_{t}=\frac{1}{\left(\zeta k_{B} T_{0} / \eta m_{p} c^{2}\right)+1} .
$$

Using (23) for $n_{B}(\tau)$ at time $t=\tau$ and (27) for $g_{0}$ we can solve for $f_{m}$,

$$
f_{m}=\left(\frac{n_{B} m_{p}}{g_{t}}\right)\left(\frac{16 \pi G \tau^{2}}{3}\right)=2 \Omega_{B}\left[\left(\zeta k_{B} T_{0} / \eta m_{p} c^{2}\right)+1\right],
$$

where $n_{B}=n_{B}(\tau)$ and $\Omega_{B}=m_{p} n_{B} / \rho_{c}$.

\section{Conclusion}

We have shown that CSR is quite adequate for a development of a nuclear physical analysis of the very early moments of the universe, from $10^{-3} \mathrm{sec}$. to $10^{3} \mathrm{sec}$. The modeling assumes two possible final temperatures for the universe, $T_{\text {past }} \approx 14.42 \mathrm{~K}$, which is the temperature the universe would attain at the present epoch if there were no recombination at time $t c \approx 1.98 \times 10^{13} \mathrm{sec}$, and $T_{0} \approx 2.73 \mathrm{~K}$, which is the present $\mathrm{CMB}$ temperature including the recombination period when the temperature was about $0.3 \mathrm{eV}(3500 \mathrm{~K})$. The details of the transition in temperature at the recombination period are not covered in this report, but we should expect some modifications to the temperature profile when that analysis is carried out.

Consider the neutron to proton ratio using CSR based on [4], Equation (1),

$$
\left(\frac{n}{p}\right)=\exp \left(\frac{-Q}{T}\right)
$$

where $n$ is the number of neutrons, $p$ is the number of protons,

$Q=m_{n}-m_{p}=1.293 \mathrm{MeV}$, where $m_{n}$ and $m_{p}$ are the masses of the neutron and proton, respectively, and $T$ is the temperature in $\mathrm{MeV}$. The neutron mean lifetime $\tau_{n} \approx 889.1 \mathrm{sec}$. (Hikasa, et al. 1992.) Figure 4 is a plot of the neutron to proton ratio using (29).

Substituting for temperature $T$ as a function of time $t$ from (20) into (29) yields the neutron to proton ratio as a function of time,

$$
\left(\frac{n}{p}\right)=\exp (-a Q \sqrt{t})
$$


where $a=(3.363)^{1 / 4} /(2.4255)^{1 / 2}$. Figure 5 is a plot of the neutron to proton ratio using (30).

The relativistic mass density $\rho_{S}(T)$ of (15) along with (16) is equivalent to the

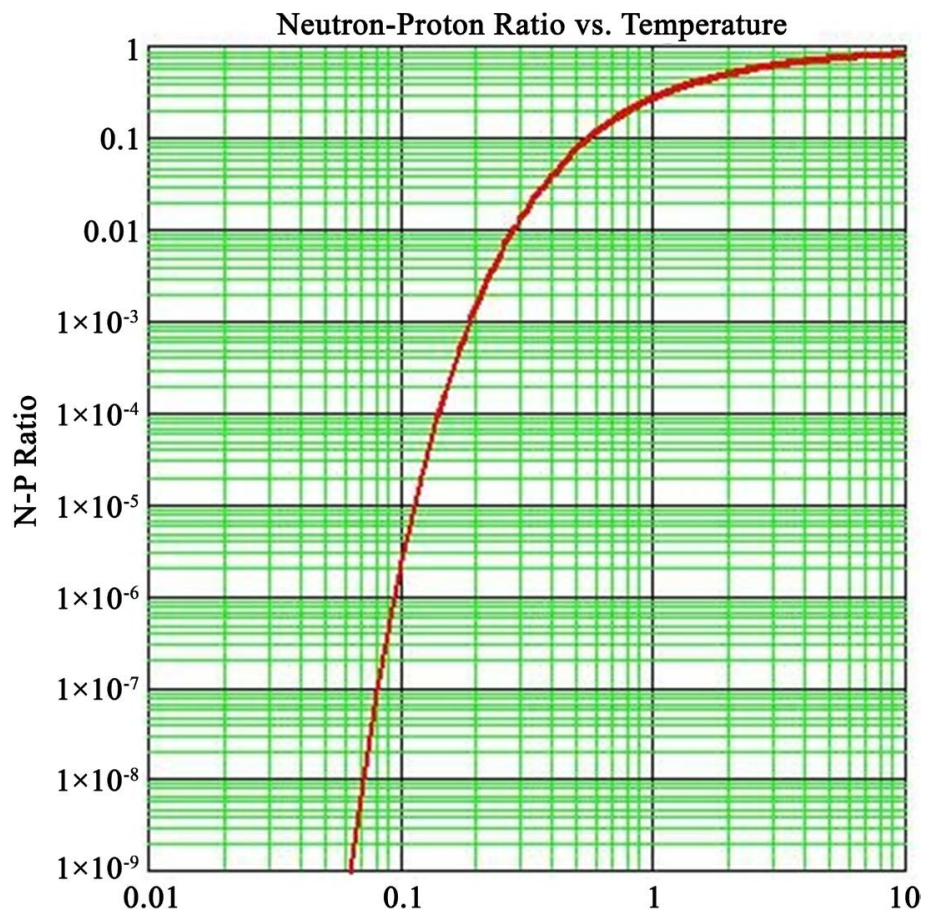

Figure 4. Neutron to proton ratio vs. temperature. The horizontal axis is the temperature in $\mathrm{MeV}$.

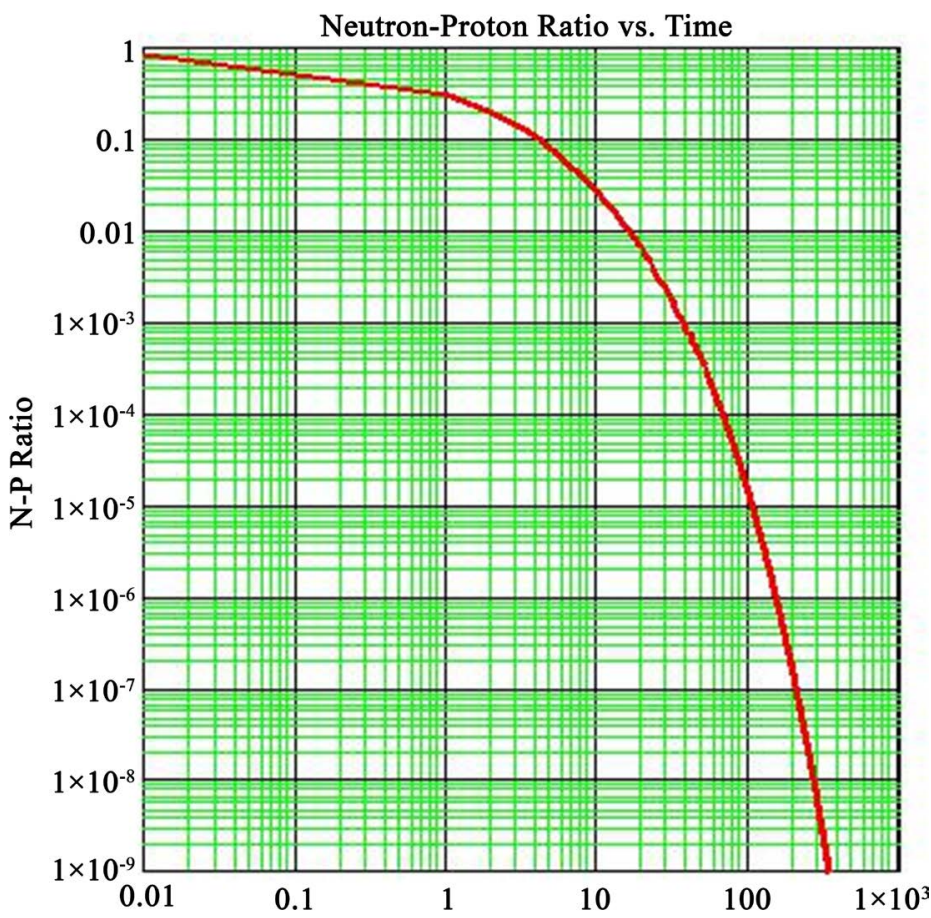

Figure 5. Neutron to proton ratio vs. time. The horizontal axis is time in seconds since the big bang. 
energy density given by Eq. (22.41) in [8]. This determines that the temperature $T \approx 1 \mathrm{MeV}$ at time $t \approx 1 \mathrm{sec}$., shown in Figure 3 , is as required for the synthesis of the light elements [9], Sect. 23.1. Thus, Carmeli's CSR theory, taking into account the internal degrees of freedom of the particles involved in the processes, has a well suited framework to describe the details of primordial nucleosynthesis.

Using (4), the rate of acceleration of the expanding universe is given by the time rate of change of the Hubble parameter $H(t)$, defined in (22), and is given by

$$
\dot{H}(t)=\frac{\ddot{R}(t)}{R(t)}-H^{2}(t),
$$

where ${ }^{\cdot}=\mathrm{d} / \mathrm{d} t$.

The standard Lambda-Cold-Dark-Matter model [10] [11], indicates that the rate of the expansion has been accelerating from around 10 billion years after the big bang when the redshift was $z \approx 0.4$. This is in contrast to the CSR model. Figure 6 is shown the acceleration $\dot{H}(t)$ from (31) for present cosmic times. We note that the acceleration remains negative (deceleration) from the big bang era up to the present. There is no need for the concept of dark matter and dark energy in the CSR model of the universe [12] [13].

A plot of $\Omega(t)$ for the present times from around $2.26 \times 10^{9}$ years up to $13.6 \times$ $10^{9}$ years since the big bang is shown in Figure 7. The density decreases through unity and ends up at the current value of $\Omega_{0} \approx \Omega_{B} \approx 0.046$. Figure 8 is

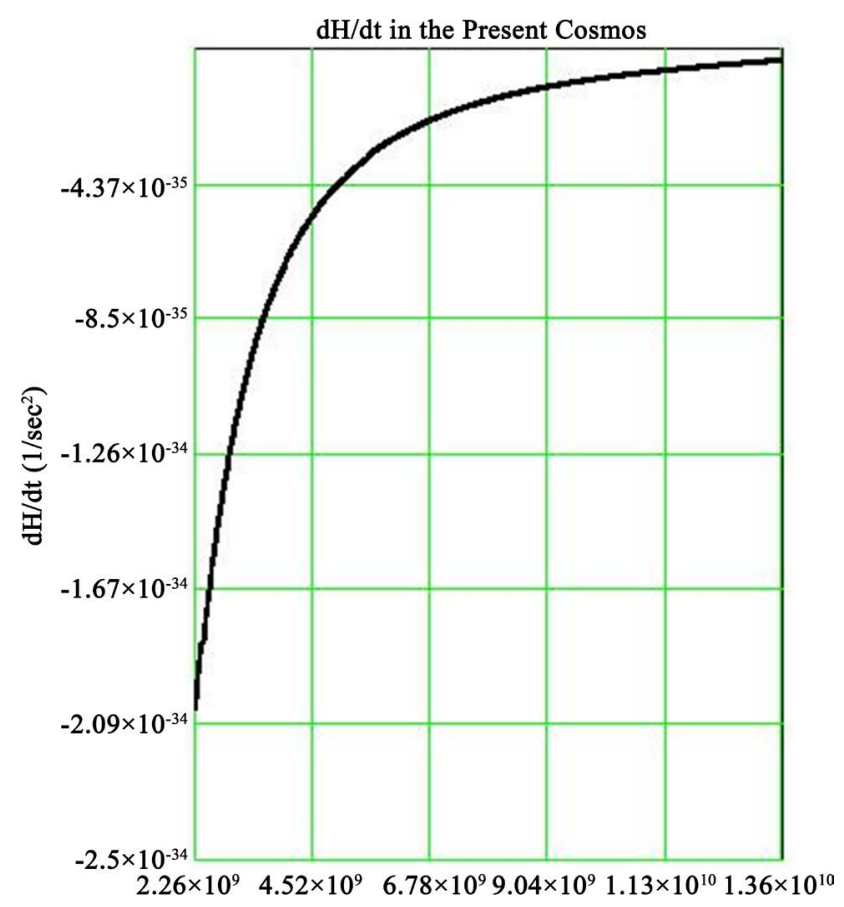

Figure 6. $\mathrm{d} H / \mathrm{d} t$ in the present cosmos from 2.26 billion years up to the present time of 13.6 billion years since the big bang. The acceleration is negative (decelerating) from the big bang to the present. The horizontal axis is time in years since the big bang. 


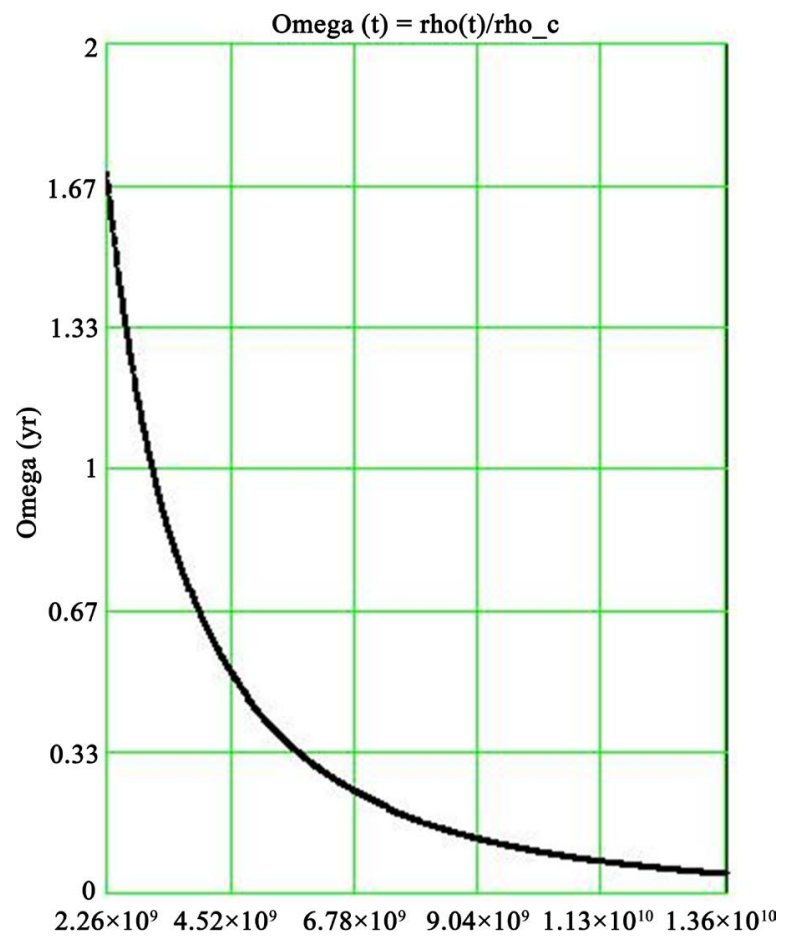

Figure 7. Total mass density parameter Omega(t) in the current cosmos from around 2.26 billion years up to the present time of 13.6 billion years since the big bang. The horizontal axis is time in years since the big bang.

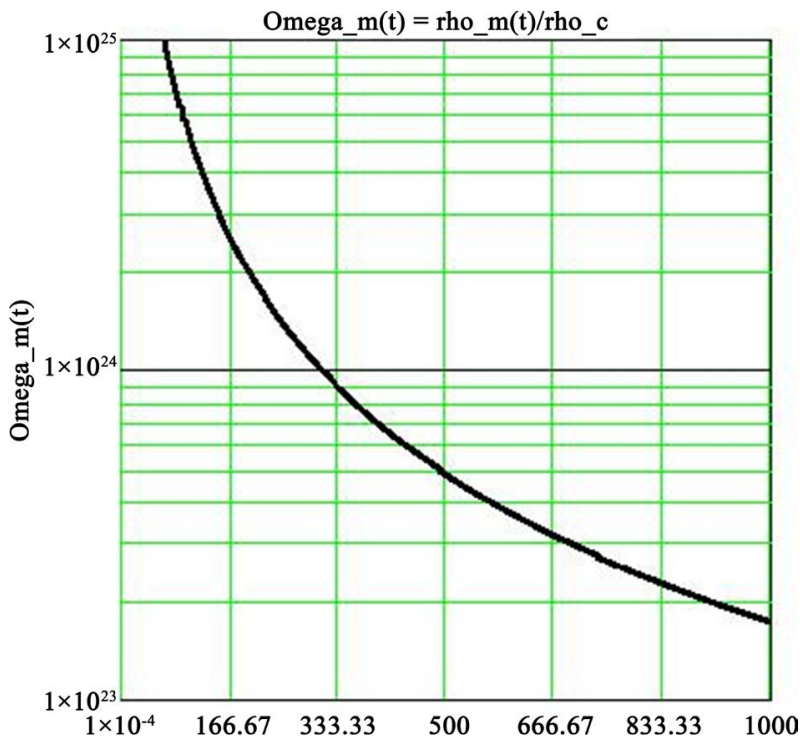

Figure 8. Omega_m(t) during the big bang nucleosynthesis period. The horizontal axis is time in seconds since the big bang.

shown the matter density parameter from (12), $\Omega_{m}(t)=\rho_{m}(t) / \rho_{c}$, for the first 1000 seconds since the big bang.

\section{Conflicts of Interest}

The author declares no conflicts of interest regarding the publication of this paper. 


\section{References}

[1] Behar, S. and Carmeli, M. (2000) Cosmological Relativity: A New Theory of Cosmology. International Journal of Theoretical Physics, 39, 1375-1396. https://doi.org/10.1023/A:1003651222960, https://arxiv.org/astro-ph/000835

[2] Carmeli, M. (2006) Cosmological Relativity. World Scientific, Singapore. https://doi.org/10.1142/6275

[3] Carmeli, M. (2008) Relativity: Modern Large-Scale Spacetime Structure of the Cosmos. World Scientific, Singapore. https://doi.org/10.1142/6820

[4] Smith, M.S., Kawano, L.H. and Malaney, R.A. (1993) Experimental, Computational and Observational Analysis of Primordial Nucleosynthesis. The Astrophysical Journal Supplement Series, 85, 219-247. https://doi.org/10.1086/191763

[5] Oliveira, F.J. (2016) Cosmic Time Transformations in Cosmological Relativity. Journal of High Energy Physics, Gravitation and Cosmology, 2, 253-279. https://doi.org/10.4236/jhepgc.2016.22022, https://file.scirp.org/pdf/JHEPGC_2016041914511433.pdf

[6] Kurki-Suonio, H. (2018) Thermal History of the Early Universe in Cosmology I, Lecture notes fall 2018.

https://www.courses.physics.helsinki.fi/teor/cosmology/Cosm4.pdf

[7] Weinberg, S. (2008) Cosmology. Oxford University Press, New York.

[8] Olive, K.A. and Peacock, J.A. (2014) Big-Bang Cosmology. Chinese Physics C, 38, 327-338.

[9] Fields, B.D., Molaro, P. and Sarkar, S. (2014) Big-Bang Nucleosynthesis. Chinese Physics C, 38, 339-344. https://arxiv.org/pdf/1412.1408.pdf

[10] Riess, A.G., Strolger, L.-G., Casertano, S., Ferguson, H.C., Mobasher, B., et al. (1998) Observational Evidence from Supernovae for an Accelerating Universe and a Cosmological Constant. Astronomical Journal, 116, 1009.

https://doi.org/10.1086/300499

[11] Frieman, J.A., Turner, M.S. and Huterer, D. (2008) Dark Energy and the Accelerating Universe. Annual Review of Astronomy and Astrophysics, 46, 385-432. https://doi.org/10.1146/annurev.astro.46.060407.145243

[12] Krizek, M. and Somer, L. (2014) A Critique of the Standard Cosmological Model. Tutorial Neural Network World, 24, 435-461.

https://dx.doi.org/10.14311/NNW.2014.24.026

[13] Krizek, M. (2018) Ten Arguments Against the Proclaimed Amount of Dark Matter. Gravitation and Cosmology, 24, 350-359.

https://dx.doi.org/10.1134/S020228931804014X 